Int. J. Dev. Biol. 54: 203-208 (2010)

doi: $10.1387 / \mathrm{ijdb} .082743 \mathrm{ys}$

\title{
Identification and expression of ventrally associated leucine-zipper (VAL) in Xenopus embryo
}

\author{
YUKO SAITO', YUHTA TAKAHASHI ${ }^{1}$, YUMI IZUTSU² and MITSUGU MAÉNO*,2 \\ ${ }^{1}$ Graduate School of Science and Technology and \\ ${ }^{2}$ Department of Biology, Faculty of Science, Niigata University, Niigata, Japan
}

\begin{abstract}
In the present study, we have isolated a novel gene that is specifically expressed in the ventral region of Xenopus neurula and tailbud embryos. This gene, referred to as ventrally associated leucine-zipper (val), encodes for a novel class of protein consisting of a leucin-zipper motif, a glutamic acid-rich sequence and 5 repeats of proline-rich sequence. Expression of val started at the mid-gastrula stage, peaked at the early tailbud stage, and disappeared by the end of tailbud stage, and the endogenous expression of val was strictly dependent on BMP signaling. Myc-tagged val protein injected at early stage was accumulated in the nucleus at the gastrula stage and later, suggesting involvement of val in the process of ventral tissue formation during the neurula and tailbud stages.
\end{abstract}

KEY WORDS: BMP, ventral expression, nuclear factor, leucine-zipper, frog

In vertebrate embryos, BMP signaling is essential for development of the ventral tissues, such as the blood island mesoderm (Dale et al., 1992; Jones et al., 1992; Maéno et al., 1994; Fainsod et al., 1994; Maéno et al., 1996; Dosch et al., 1997; Mead et al., 1998; Connolly et al., 2000). Extensive studies have demonstrated that several transcription factors at downstream of the BMP signaling are involved in the differentiation of blood and endothelial lineages in frog embryo (Kelly et al., 1994; Walmsley et al., 1994; Mead et al., 1998; Friedle and Knöchel, 2002), but the differentiation program of primitive ventral blood islands is not fully understood (Kikkawa et al., 2001; Maeno, 2003). Thus, we made a subtracted cDNA library from early neurula embryos to isolate genes that were specifically expressed in the ventral region and involved in the development of ventral organs (Shibata et al., 2008). The present study identifies a novel gene that is expressed in the ventral part of neural and tailbud embryos. This gene is expressed in the BMP signaling-dependent manner and is coding for a novel class of nuclear factor that consists of predicted protein interaction domains; i.e. a leucine zipper and repeated proline-rich domains.

\section{Isolation of a cDNA clone that is associated with ventral tissue formation}

We have identified cDNA clones preferentially expressed in the ventral and lateral regions of Xenopus neurula embryo by a
PCR-based subtraction method to concentrate cDNAs derived from the BMP signal-activated embryos (Shibata et al., 2008). Approximately $200 \mathrm{cDNA}$ clones from the subtracted library were sequenced and further screened by whole-mount in situ hybridization analysis to select the cDNAs that were preferentially expressed in ventral part of the embryos at the neurula stage. Transcript of one cDNA clone (cl. 162) was strongly expressed in ventral part of the neurula embryo but not in the other regions. Thus, we characterized this clone further. We isolated a phage clone from the ZAPII library. Sequence analysis showed that this clone consisted of 1,654 nucleotides, and it encoded for a protein of 454 amino acids.

Nucleotide sequences showed that this CDNA encodes for a novel protein containing a leucine zipper motif and 5 repeats of proline-rich sequences and a glutamic acid-rich domain (Fig. 1A). Homology search against the DNA databases indicated that Xenopus tropicalis has a similar gene in the genome database. The predicted amino acid sequence of $X$. tropicalis counterpart contains a nuclear localization signal (NLS), leucine-zipper motif and proline-rich repeats (Fig. 1B). The direct comparison of nucleotide sequences in the 5 '-end region of DNA including the

\footnotetext{
Abbreviations used in this paper: BMP, bone morphogenetic protein; VAL, ventrally associated leucine-zipper.
}

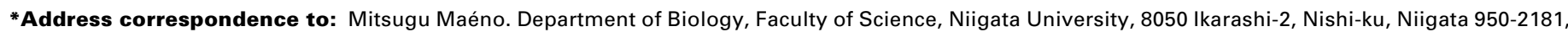
Japan. Fax+81-25-262-6116. e-mail: maenobio@bio.sc.niigata-u.ac.jp
}

Supplementary Material for this paper (one figure) is available at: http://dx.doi.org/10.1387/ijdb.082743ys

Final author corrected PDF published online: 2 October 2009.

ISSN: Online 1696-3547, Print 0214-6282 
A

actggattatagctttcctctcttctcctgaaccagtgaat ttgctatctaccgcagctccttcctataacataATGtacagctcagacgaagaggattt $\begin{array}{lllllllll}M & Y & S & S & D & E & E & D & F\end{array}$ L N I E E K N F F L N Q A M Q A V L R T N $\begin{array}{cccccccccccccccccccc}\text { L } & \text { N } & \text { I } & \text { E } & \text { E } & \text { K } & \text { N } & \text { F } & \text { F } & \text { L } & \text { N } & \text { Q } & \text { A } & \text { M } & \text { Q } & \text { V } & \text { L } & \text { R } & \text { T } & \text { N } \\ \text { gttgcacagatagaagaaagcatagataatgtaaataaagaactcaagaaacatgaatat }\end{array}$ $\begin{array}{lllllllllllllllllllll}V & \text { A } & Q & \text { I } & \text { E } & \text { E } & \text { S } & \text { I } & \text { D } & \text { N } & \text { V } & \text { N } & \text { K } & \text { E } & \text { L } & \text { K } & \text { K } & \text { H } & \text { E } & \text { Y } & 4\end{array}$ gacctcaaact tagtcacaatgagctaaaaagctgaaagaagaagaagaaaccctgcaa

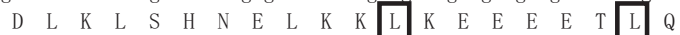
ctggaagctgaaacattagaagaacagctgcagaccctgaataaagagagtgagttgcta $\begin{array}{lllllllllllllllllllll}\text { L } & E & A & E & T & L & E & E & Q & L & Q & T & L & N & K & E & S & E & L & L & 89\end{array}$ gagaaaaatatgcagacatgtatagaatagttaaaagcataagaactcttggaccaata

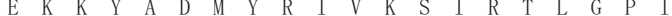

tttcaacaagcaatggcagtcacactcagtaacatgaatgcaataataaacacgatgtct $\begin{array}{lllllllllllllllllllll}\text { F } & \text { Q } & \text { Q } & \text { A } & \text { M } & \text { A } & \text { V } & \text { T } & \text { L } & \text { S } & \text { N } & \text { M } & \text { N } & \text { A } & \text { I } & \text { I } & \text { N } & \text { T } & \text { M } & \text { S } & 129\end{array}$ caagaaggcattatcggtcttcagccetgcacagacccgaatcagaatctttaccaagg $\begin{array}{llllllllllllllllllll}\text { Q } & E & G & I & I & G & L & Q & P & C & T & D & P & E & S & E & S & L & P & R\end{array}$ acccaagtgccatctgcctctgaaagtttgcccgaagtccagcaaaaatcattgtcaatc $\begin{array}{lllllllllllllllllllll}T & Q & V & P & \text { S } & A & S & E & S & L & P & E & V & Q & Q & K & S & L & S & I & 169\end{array}$ accccagtgccacctgectctgaaaccttgcaat tggtccaaccaaaagggacccctgtg $\begin{array}{llllllllllllllllllll}\text { T } & \text { P } & \text { V } & \text { P } & \text { P } & \text { A } & \text { S } & \text { E } & \text { T } & \text { L } & \text { Q } & \text { L } & \text { V } & \text { Q } & \text { P } & \text { K } & \text { G } & \text { T } & \text { P } & \text { V }\end{array}$ ccatctacctctaaaagctttcccaaagtacagccaaaatctttgccaagcaccccattg

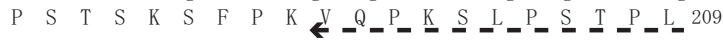
tcatctgcttctgaaagattgcccacagtccaaccaaagtatttgccaattaccccagtg

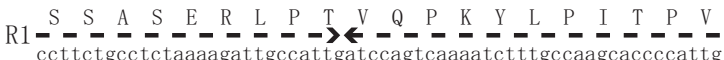

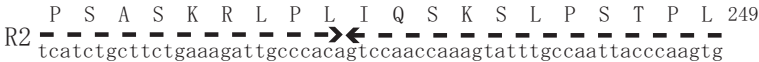

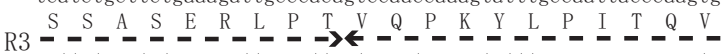
ccttctgcotctaaaagat tgccattggtccagtcaaaatetttgccaagcaccccastg

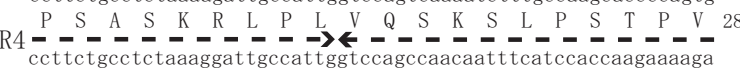
$\begin{array}{lllllllllllllllllllll} & \text { P } & S & A & S & K & G & L & P & L & V & Q & P & T & I & S & S & T & K & K & R\end{array}$ R5 -

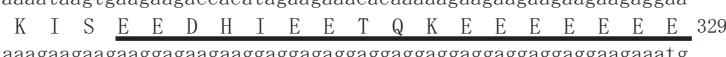
\begin{tabular}{llllllllllllllllllll}
$K$ & $E$ & $E$ & $E$ & $G$ & $E$ & $E$ & $G$ & $G$ & $E$ & $E$ & $E$ & $E$ & $E$ & $E$ & $E$ & $E$ & $E$ & $E$ & $M$ \\
\hline
\end{tabular} atggaagaatttgacacaaaaacagacacagctaccaaactgcctccatttcttcttcca $\begin{array}{lllllllllllllllllllll}\text { M } & \text { E } & \text { E } & \text { F } & \text { D } & \text { T } & \text { K } & \text { T } & \text { D } & \text { T } & \text { A } & \text { T } & \text { K } & \text { L } & \text { P } & \text { P } & \text { F } & \text { L } & \text { L } & \text { P } & 369\end{array}$ tatataccaaaaagaaatctccgaatgataagtggecccttaactatacagcttttctt Y aattattactttgaaacaacttttaaaggaaagctttcaaaagaggttgtactggattgc $\begin{array}{lllllllllllllllllllll}\mathrm{N} & \text { Y } & \text { Y } & \mathrm{F} & \mathrm{E} & \mathrm{T} & \mathrm{T} & \mathrm{F} & \mathrm{K} & \mathrm{G} & \mathrm{K} & \mathrm{L} & \mathrm{S} & \mathrm{K} & \mathrm{E} & \mathrm{V} & \mathrm{V} & \mathrm{L} & \mathrm{D} & \mathrm{C} & 409\end{array}$

attcagcagggtataaactataatattteccettaaaacaatccaatgataaactgtac $\begin{array}{lllllllllllllllllllll} & \mathrm{Q} & \mathrm{Q} & \mathrm{G} & \mathrm{I} & \mathrm{N} & \mathrm{Y} & \mathrm{N} & \mathrm{I} & \mathrm{F} & \mathrm{P} & \mathrm{L} & \mathrm{K} & \mathrm{Q} & \mathrm{S} & \mathrm{N} & \mathrm{D} & \mathrm{K} & \mathrm{L} & \mathrm{Y}\end{array}$ aaacaagtttatacaaggattgtccatttaaaaaggcaaagggaagggatgaagaaaaga $\begin{array}{lllllllllllllllllllll}\text { K } & \text { Q } & \text { V } & \text { Y } & \text { T } & \text { R } & \text { I } & \text { V } & \text { H } & \text { L } & \text { K } & \text { R } & \text { Q } & \text { R } & \text { E } & \text { G } & \text { M } & \text { K } & \text { K } & \text { R } & 449\end{array}$ tacaacaggagacagTAAtagaatcaaagacataaaaacccacatattacctctgtcccc $\begin{array}{llllllllll} & \mathrm{N} & \mathrm{N} & \mathrm{R} & \mathrm{R} & \mathrm{Q} & *\end{array}$

ttttccttgttctgcagtgaaagtagggaccacatttgtttgggatatattattccctac tagttaccttttatgataaatgattcctgtgtagtatttctttgtttctaatgtacact actcttattgttgcactAATAAAacttttaaaaataaaaaaaaaaaaaaaaa

predicted first methionine between these two species indicated that these regions are highly conserved each other (Fig. 1C). No information of protein possessing the related structure is available in other vertebrate species, except a partial correlation of the proline-rich sequences was found.

\section{Expression of val (cl. 162) transcripts in embryo and adult tissues}

Northern blot analysis revealed that a transient expression of cl. 162 mRNA was observed in a period between neurula and tailbud stages. The size of mRNA was estimated to $\sim 1,720$ bases in comparison with loading distances of the RNA ladder markers. We concluded that the cDNA clone, therefore, covers almost entire sequence of mRNA. We examined the expression of $\mathrm{cl} .162$ in various adult organs, such as brain, muscle, heart and others, but none of organ expressed any trace of transcript (Fig. 2B). The

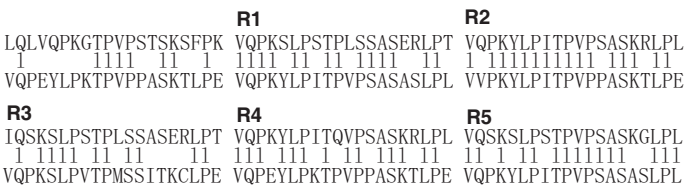

Fig. 1. The primary structure of valcDNA

(A) Nucleotide and deduced amino acid sequences of val. Fulllength cDNA clone (cl. 162) encodes for an open reading frame consisting of 454 amino acids. This protein has a leucine zipper region (squares), 5 repeats of proline-rich sequences (dotted lines, R1-R5) and a glutamic acid-rich sequence (underline). There is a polyadenylation signal sequence (capitalized) in the 3' UTR. (B) Comparison of proline-rich D E repeat sequences of Xenopus laevis val and Xenopus tropicalis undefined counterpart (from genome sequence), indicating that the amino acid sequences in these repeats are conserved between these two species. (C) Alignment of 5'-UTR nucleotide sequences of X. laevis and X. tropicalis val genes. The nucleotide sequences in the region near the predicted translation initiation site (ATG) are highly conserved.X. tropicalis genome sequence has a stop codon (star) at upstream region in the same frame, suggesting that this ATG is most likely the translation initiation site.

result demonstrated that $c l .162$ transcript appears highly specific in embryonic stage.

We performed in situ hybridization analysis to show the localization of $\mathrm{cl}$. 162transcript in embryogenesis. A small amount of transcript was first visible in the mid-gastrula embryo at the yolk plug region (Fig. $3 \mathrm{~A}, \mathrm{~B}$ ). This region will contribute to endodermal derivatives. At the early neurula stage, expression was observed in the ventral part of the embryo, especially in the posterior level (Fig. 3 C,D). The highest level of expression was observed in the broad region of ventral part of embryo at the neurula and early tailbud stages (Fig. $3 \mathrm{E}-\mathrm{J}$ ). The positive area included the ectoderm, mesoderm and endoderm layers (Fig. 3K,K'). The level of expression started to decrease as the stage advanced in the ventral part of embryo at the tailbud stage, and the transcript remained in the anterior and posterior regions (Fig. $3 \mathrm{~L}, \mathrm{M}$ ). At the late tailbud stage, expression of $\mathrm{cl}$. 162 was concentrated in the triangle region at the anterior ventral area (Fig. $3 \mathrm{~N}, \mathrm{O}$ ) where hex is positively expressed (Fig. $3 \mathrm{Q}, \mathrm{R}$ ). This area matched to the liver primordium but not to the blood island area (Fig. 3P) or to the heart primordium (Fig. 3S). At the swimming tadpole stage, two small spots of positive area were visible in the anterior and posterior ventral parts (Fig. $3 \mathrm{~T}, \mathrm{U}$ ). Because of this striking pattern of expression in embryogenesis, we named this gene as ventrally associated leucine-zipper (val).

\section{Regulation of val expression in response to BMP signaling}

Expression pattern of $v a l$ in the developing embryos and adult tissues suggested involvement of val in the process of ventral morphogenesis in Xenopus embryo. We analyzed how the expression of $v a l$ is regulated by basic signals that govern the 
embryonic axis formation. As expected, message of val was significantly enhanced by BMP-4 mRNA injection (2ng/embryo) in the presumptive dorsal blastomeres at the 4 cell-stage. Wholemount in situ hybridization showed that the expression of val expanded in the ventral and lateral regions in the BMP-4-injected embryos at the tailbud stage (stage 23) (Fig. 4 A,B) $(n=12$ for control and $n=12$ for $B M P-4$ injection). In contrast, injection of $t B R$ RNA (coding for a dominant-negative form of BMP-4 receptor, 1.5 ng/embryo) down-regulated the expression of $v a l$ in the resultant embryos (Fig. 4C) ( $n=11$ for $t B R$ injection). Semi-quantitative RTPCR analysis was also performed using the ventral (VMZ) and dorsal (DMZ) marginal zone explants to show the expression of val in $t B R$ RNA-injected tissues (Fig. 4D). The ventral markers such as vent-1 and gata-2 were highly expressed in the VMZ explants at stage 20 and val was also detected in the VMZ explants. These markers were less expressed in the control DMZ explants. When $t B R$ RNA (1.5 ng/embryo) was injected in the ventral marginal zone at the 4-cell stage, expression of val as well as the other ventral markers was suppressed. Instead, nrp-1, a neural marker, was induced in the $t B R$-injected $\mathrm{VMZ}$ explants. These results demonstrate that the BMP-4 signal is necessary and sufficient for the endogenous val expression. In order to examine whether downstream genes of the BMP signal can activate the expression of valin embryonic cells, we overexpressed

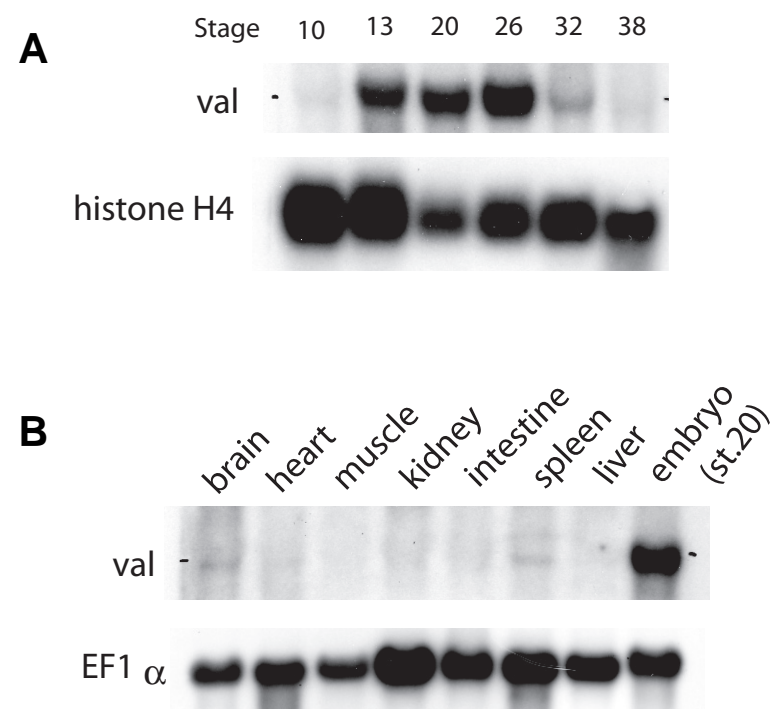

the vent-1 (1 ng/embryo), gata-1 (0.25 ng/embryo) or gata-2(2 ng/ embryo) mRNA in the DMZ at the 4-cell stage and the DMZ explants were cultured until stage 20 . The RT-PCR analysis

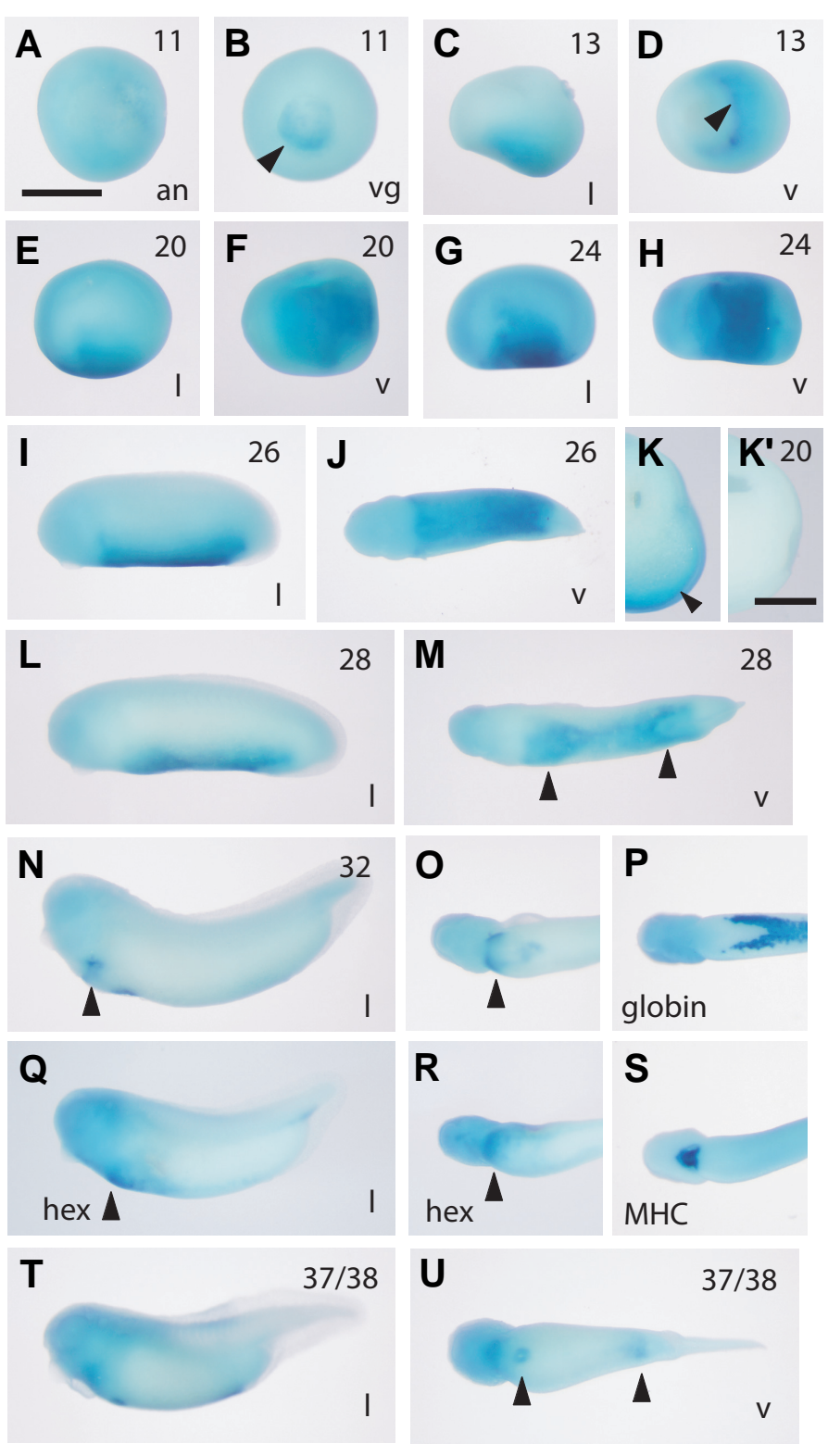

Fig. 2 (Left). Northern analysis of val in developing embryos and adult organs. Total RNA from the embryos at different stages (A) or the adult organs (B) were extracted and purified by AGPC method (Chomczynski and Sacchi, 1987) and electrophoresed on a 1\% agarose gel. Blots were hybridized with the ${ }^{32}$ P-dCTP-labelled val probes. After dehybridization, the same blots were hybridized with histone $\mathrm{H} 4$ or ef1 $\alpha$ probe. A single band at approximately $1.6 \mathrm{~kb}$ was detected in the developing embryos between st. 13 and st. 32 (late gastrula and tailbud stages). No obvious signal was detected in adult organs ever examined.

Fig. 3 (Right). Whole-mount in situ hybridization analysis of valin developing embryos. A very faint expression of val message was first detected in the yolk plug (presumptive endoderm) at the mid-gastrula stage (st. 11) (A,B). Expression was gradually accumulated in the posterior ventral part of the neurula embryo (st. 13-20) (C-F) and peaked at the early tailbud stage (st. 24) (G,H). Dissection of the stained embryo (st. 20) showed that val was expressed in all three germ layers (K). K' shows a control dissected embryo hybridized with the sense probe. During the tailbud stages, the expression of val extended toward anterior and posterior directions (st. 26-28) (I,J,L, $\mathbf{M})$ and two domains of expression areas were separated each

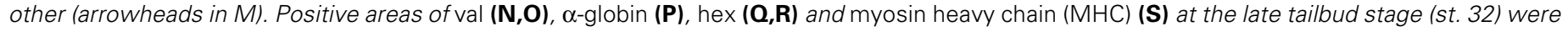
compared each other, suggesting that val-positive area overlapped with hex-positive area (a region of the liver rudiment as indicated by arrowheads in N, O, $Q$ and R). Positive messages were visible in the two spots of anterior and posterior regions (arrowheads in U) in the swimming tadpoles (st. 37/38) (T,U). Scale bars in A and $\mathrm{K}^{\prime}$ show $1 \mathrm{~mm}$ and $500 \mu \mathrm{m}$, respectively. 


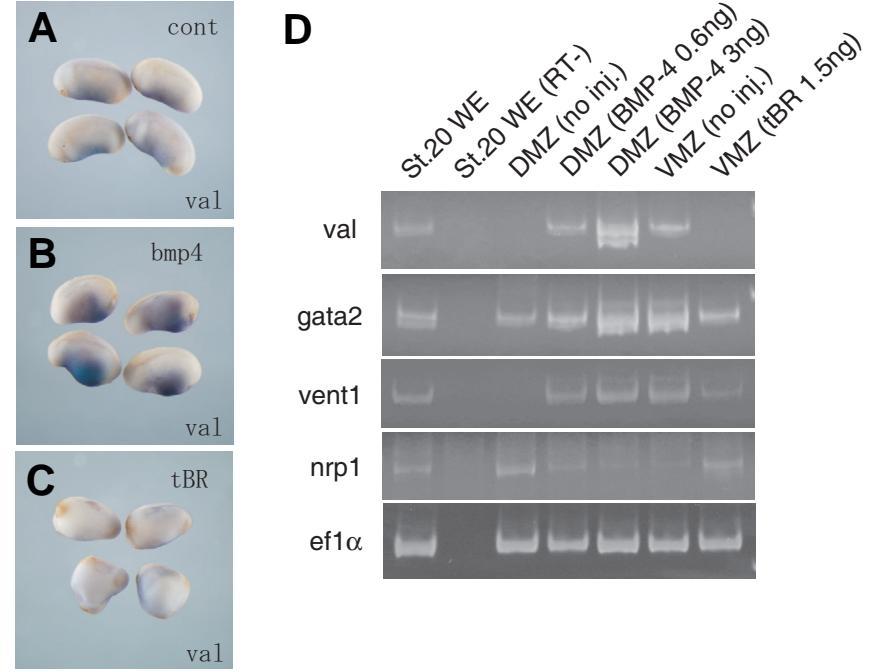

Fig. 4. Regulation of val expression by the BMP signal. (A-C) Embryos were injected with 2ng BMP-4 (B) or $1.5 \mathrm{ng}$ tBR (C) RNA and cultured until the early tailbud stage (st. 23). Uninjected control embryos were also cultured (A). These embryos were fixed and subjected to the wholemount in situ hybridization analysis for detection of val mRNA. (D) Dorsal marginal zone (DMZ) or ventral marginal zone (VMZ) explants were prepared from the embryos that had been injected with BMP-4 10.6 or 3 ng) or tBR (1.5 ng) RNA at the 4-cell stage and cultured until the early tailbud stage (st. 20). Explants were subjected for the RT-PCR analysis to detect the expression of val, vent-1, gata-2, nrp-1 and ef1 $\alpha$. Note that val expression is essentially depending on the BMP signal.

showed that none of these factors enhanced the expression of $v a l$ as BMP-4 mRNA did (data not shown), suggesting a complex regulation may be involved in the initiation of val expression.

\section{Myc-tagged val protein is localized in nucleus at the gastrula stage}

In order to speculate function of val in embryonic cells, we examined the subcellular localization of val protein by expressing myc-tagged protein in embryonic cells. As we mentioned above, the most related predicted protein in X. tropicalis has a NLS at the $\mathrm{N}$-terminal region, although val has no typical NLS sequence. We injected RNAs coding for myc-val and myc-mif proteins in the two blastomeres at the 4-cell stage, and localization of the exogenous proteins was analyzed. Mif is a cytosolic protein that was previously published (Suzuki et al., 2004). Western blot analysis indicated that both val and mif proteins were efficiently expressed in the embryonic cells as expected (Fig. 5A). Subsequent wholemount immunostain experiment showed that both myc-val and myc-mif were present in the cytoplasm at st. 8 (early blastula stage) (Fig. $5 \mathrm{~B}, \mathrm{D}$ ), but myc-val became localized in the nucleus at st. 12 (gastrula stage) (Fig. $5 \mathrm{C}$ ) whereas myc-mif was still present ubiquitously (Fig. 5E). In order to confirm nuclear localization of val protein, antibody-stained embryos at st. 12 were sectioned and counter-stained with Hoechst 33258. As shown in Fig. $5 \mathrm{~F}, \mathrm{G}$, val-positive regions coincided with the positions of nuclei. This result demonstrates that val has a NLS and is transported into nucleus at the stage at which endogenous val is expressed in the embryonic cells.

The most important information in characterization of val is its striking expression pattern in embryogenesis. Highly specific expression of val message at the ventral part of the embryo suggests involvement of val in the process of ventral tissue formation. The present study also demonstrated that the expression of valdepends the existence of the BMP signal, but we think that, unlike gata-2, val is not a direct target of BMP, because val starts to express in the embryo later than gata-2, which is expressed at early gastrula stage (Kelly et al., 1994; Walmsley et al., 1994). We also examined whether wnt signal controls the expression of $\mathrm{val}$ in the embryonic cells. As shown in Fig. S1 A$\mathrm{C}$, injection of $d k k 1$ induced an anteriorizing phenotype in the embryo. However, the RT-PCR analysis revealed that amount of $\mathrm{val}$ message is not significantly enhanced by $d k \mathrm{k} 1$ injection (Fig. S1D), indicating that endogenous expression of $v a l$ is independent of the canonical wnt signaling pathway. Therefore, it is likely that val is a factor located at downstream of the BMP signaling specifically.

The sequence analysis demonstrates that val encodes for a novel class of protein that contains a putative leucine-zipper motif and 5 repeats of proline-rich region. The proline-rich region picked up some uncharacterized proteins (Q9JJ89 or A5UTR1) but with a low homology (20-30\% identical). Synteny analysis using Metazome program (JGl) did not hit any equivalent gene in mammalian genomes. This suggests that val does not take part

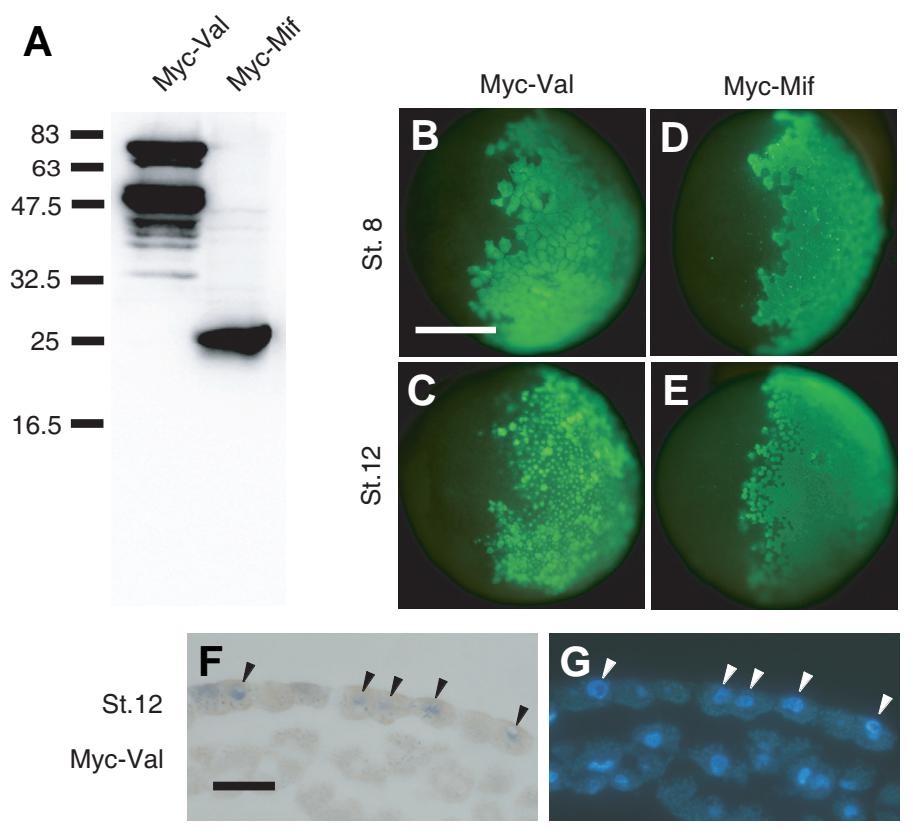

Fig. 5. Localization of val protein in nucleus at the gastrula stage. (A) Biochemical detection of myc-tagged val and mif (Suzuki et al., 2004) by Western blot analysis. Embryos were injected with myc-val and myc-mif RNA (4ng/embryo) into two blastomeres at the 2-cell stage, and they were allowed to develop until st. 24. Soluble proteins extracted from these embryos were loaded in $12.5 \%$ SDS-PAGE. Anti-myc antibodypositive bands were visualized with a peroxidase-based chemi-luminescence substrate. (B-G) Whole-mount immunostaining shows subcellular localization of myc-val (B,C) and myc-mif $(\mathbf{D}, \mathbf{E})$ proteins at st. $8(B, D)$ or st. $12(C, E)$. (F, G) Section through the immunostained embryo shows the localization of myc-val protein (F) and nuclei by Hoechst staining (G). Note that myc-val protein is localized in the nucleus (arrowheads in Fand G) at the gastrula stage, but not at the early blastula stage. Scale bars indicate $500 \mu \mathrm{m}(B)$ and $50 \mu \mathrm{m}(F)$, respectively. 
in the conserved cascade of tissue specification process in development. However, stage-specific localization of val protein in the nucleus (Fig. 5) and spatial distribution of this protein (Fig. 3) evoke an idea that this protein may function together with essential molecules in determination and specification of the ventral tissues, such as blood islands and digestive organs. Functional analyses of val using mutant constructs as well as antisense morpholinos are undertaken.

\section{Materials and Methods}

\section{Isolation of val from the embryonic cDNA library}

Subtraction of cDNA made from the BMP-4-injected embryos was performed with a PCR-Select ${ }^{\mathrm{TM}}$ cDNA Subtraction Kit according to the protocol of the manufacturer (BD Biosciences) using poly $(A)+R N A$ from st. 10 embryos as a driver. Subtracted cDNAs were cloned into the T-Easy vector (Promega), and randomly selected clones were sequenced and a part of them with novel sequences were further analyzed by the wholemount in situ hybridization analysis. A full-length cDNA clone was isolated from the st. 18 Uni-ZAPII library. The nucleotide sequence of val cDNA has been deposited in the DDBJ database (AC\#AB432918).

\section{Embryos and microinjection}

Xenopus laevis females were injected with 125-250 units of human chorionic gonadotropin and kept at $23^{\circ} \mathrm{C}$ overnight to induce ovulation. Fertilized eggs were dejellied in $2.5 \%$ thioglycolic acid ( $\mathrm{pH} 8.3$ ) solution for a few minutes. After washing, the embryos were cultured in $100 \%$ Steinberg's solution ( $58 \mathrm{mM} \mathrm{NaCl}, 0.7 \mathrm{mM} \mathrm{KCl}, 0.5 \mathrm{mM} \mathrm{Ca}\left(\mathrm{NO}_{3}\right)_{2}, 0.85$ $\mathrm{mM} \mathrm{MgSO}$, $4.6 \mathrm{mM}$ Tris- $\mathrm{HCl}, \mathrm{pH}$ 7.4) until use. Developmental stages were determined as described by Nieuwkoop and Faber (1994).

Capped mRNAs for microinjection were synthesized according to the protocol of the manufacturer (Megascript, Ambion). BMP-4 and $t B R$ in pSP64T, dkk1 in pCS2+, vent-1 in pBS, and gata-1 and gata-2 in pGEM$\mathrm{HE}$ were linearized by the appropriate enzyme and RNA was transcribed with SP6 or T7 polymerase. Activation of wnt signal was achieved by injection of wnt8DNA in pCSKA. RNA or DNA was injected into the animal pole area of 2-cell-stage embryos or into the ventral marginal zone or dorsal marginal zone of 4-cell-stage embryos in 100\% Steinberg's solution containing $3 \%$ Ficoll by using a micromanipulator (Nanoject, Drummond).

\section{Whole-mount in situ hybridization and Northern blot analyses}

Whole-mount in situ hybridization analysis was performed as described previously (Shain and Zuber, 1996). Digoxigenin-labeled antisense ribonucleotide probes were synthesized as follows: myosin heavy chain (MHC)- $\alpha$ in pBS was linearized by Sal I and RNA was transcribed with T3 polymerase; hex in pBS was linearized by BamHI and RNA was transcribed with T7 polymerase. For Northern blot analysis, RNA (10 $\mu \mathrm{g} /$ lane) from embryos and various adult tissues was run in a denatured $1 \%$ agarose gel, transferred to a nylon membrane, and hybridized with fulllength cDNA fragment of clone 162 (1.6-kb EcoRI/Xhol fragment). The membrane was rehybridized with histone $\mathrm{H} 4$ (0.5-kb PCR-amplified fragment) or ef $1 \alpha$ (0.8-kb Pstl fragment).

\section{Western blot analysis and immunostaining}

Myc-tagged val and myc-tagged mif (macrophage inhibitory factor) were constructed in pCS2+MT vector, and RNAs were transcribed with SP6 polymerase. After injection of RNA, embryos at appropriate stage were harvested and subjected for Western blot analysis or whole-mount immunostaining. For Western blot, extract was loaded in 12.5\% SDSPAGE, and transferred membrane was incubated with anti-myc antibody (9E10, Santa Cruz Biotechnology) and PO-conjugated anti-mouse IgG antibody. Positive signals were visualized by ECL ${ }^{\mathrm{TM}}$ Western Blotting Detection Reagent (GE Healthcare). For immunostaining, embryos were incubated with anti-myc antibody and Alexa-conjugated anti-mouse IgG antibody (Alexa Fluor 488, Molecular Probe) or AP-conjugated antimouse IgG antibody (Santa Cruz Biotechnology). Nuclei in some sections were counter-stained with Hoechst 33258.

\section{RT-PCR analysis}

Forward and reverse primers used in this study are as follows:

ef1 $\alpha$ (221 bp, 24 cycle),

5-cct-gaa-tca-ccc-agg-cca-gat-tgg-tg-3 and

5-gag-ggt-agt-ctg-aga-agc-tct-cca-cg-3;

val (264 bp, 30 cycle),

5-ctg-cag-acc-ctg-aat-aaa-gag-3 and

5-tga-ttt-ttg-ctg-gac-ttc-ggg-3;

gata-2 (267bp, 26 cycle),

5-gga-act-ttc-cag-gtg-cat-gca-gga-g-3 and

5-ccg-agg-tgc-aaa-tta-tta-tgt-tac-3;

vent -1 ( $157 \mathrm{bp}, 30$ cycle),

5-ttc-cct-tca-gca-tgg-ttc-aac-3 and

5-gca-tct-cct-tgg-cat-att-tgg-3;

nrp-1 (283 bp, 30 cycle),

5-ggg-ggt-ttc-ttg-gaa-caa-gc-3 and

5-tta-ctg-tgc-agg-aac-aca-ag-3;

pox2 (230 bp, 28 cycle),

5-aag-cca-agc-tga-aca-ggc-aa-3 and

5-cag-ttt-ctg-ggc-cag-ttg-tt-3.

The PCR products were loaded on 5\% PAGE.

\section{Acknowledgements}

We thank Dr. P. A. Krieg for supplying plasmids. This work was partly supported by a grant-in-aid from The Ministry of Education, Science and Culture of Japan.

\section{References}

CONNOLLY, D. J., PATEL, K., WITHINGTON, S. and COOKE, J. (2000). Effects of follistatin and BMP4 proteins on early dorso-ventral patterning in chick. Int. J. Dev. Biol. 44: 129-140.

DALE, L., HOWES, G., PRICE, B.M.J. and SMITH, J.C. (1992). Bone morphogenetic protein 4: a ventralizing factor in Xenopus development. Development 115: 573-585.

DOSCH, R., GAWANTKA, V., DELIUS, H., BLUMENSTOCK, C. and NIEHRS, C. (1997). Bmp-4 acts as a morphogen in dorsoventral mesoderm patterning in Xenopus. Development 124: 2325-2334.

FAINSOD, A., STEINBEISSER, H. and DE ROBERTIS, E.M. (1994). On the function of BMP-4 in patterning the marginal zone of the Xenopus embryo. EMBO J. 13: 5015-5025.

FRIEDLE, H. and KNÖCHEL, W. (2002). Cooperative interaction of Xvent-2 and GATA-2 in the activation of the ventral homeobox gene Xvent-1B. J. Biol. Chem. 277: 23872-23881.

JONES, C.M., LYONS, K.M., LAPAN, P.M., WRIGHT, C.V. and HOGAN, B.L. (1992). DVD-4 (bone morphogenetic protein-4) as a posterior-ventralizing factor in Xenopus mesoderm induction. Development 115: 639-647.

KELLEY, C., YEE, K., HARLAND, R. and ZON, L.I. (1994). Ventral expression of GATA-1 and GATA-2 in the Xenopus embryo defines induction of hematopoietic mesoderm. Dev. Biol. 165: 193-205.

KIKKAWA, M., YAMAZAKI, M., IZUTSU, Y. and MAÉNO, M. (2001). Two-step induction of primitive erythrocytes in Xenopus laevis embryos: signals from the vegetal endoderm and the overlying ectoderm. Int. J. Dev. Biol. 45: 387-396.

MAÉNO, M., ONG, R.C., SUZUKI, A., UENO, N. and KUNG, H.F. (1994). A truncated bone morphogenetic protein 4 receptor alters the fate of ventral mesoderm to dorsal mesoderm: Roles of animal pole tissue in the development of ventral mesoderm. Proc. Natl. Acad. Sci. USA. 91: 10260-10264.

MAÉNO, M., MEAD, P.E., KELLEY, C., XU, R.H., KUNG, H.F, SUZUKI, A., UENO, N. and ZON, L.I. (1996). The role of BMP-4 and GATA-2 in the induction and differentiation of hematopoietic mesoderm in Xenopus leavis. Blood 88: 1965- 
1972.

MAÉNO, M. (2003). Regulatory signals and tissue interactions in the early hematopoietic cell differentiation in Xenopus laevis embryo. Zool. Sci. 20: 939-946.

MEAD, P.E, KELLEY, C.M., HAHN, P.S., PIEDAD, O. and ZON, L.I. (1998). SCL specifies hematopoietic mesoderm in Xenopus embryos. Development 125 : 2611-2620.

NIUEWKOOP, P.D. and FABER, J. (1994). Normal Table of Xenopus laevis (Daudin). Garland, New York.

SHAIN, D.H. and ZUBER, M.X. (1996). Sodium dodecyl sulfate (SDS)-based whole-mount in situ hybridization of Xenopus laevis embryos. J. Biochem. Biophys. Methods. 31: 185-188.
SHIBATA, T., TAKAHASHI, Y., TASAKI, J., SAITO, Y., IZUTSU, Y. and MAÉNO $M$. (2008). A role of D-domain-related proteins in differentiation and migration of embryonic cells in Xenopus laevis. Mech. Dev. 125: 284-294.

SUZUKI,M., TAKAMURA, Y., MAÉNO, M., TOCHINAI, S., IYAGUCHI, D., TANAKA I., NISHIHIRA and J., ISHIBASHI, T. (2004). Xenopus macrophage migration inhibitory factor is essential for axis formation and neural development. J. Biol. Chem. 279: 21406-21414.

WALMSLEY, M.E., GUILLE, M.J., BERTWISTLE, D., SMITH, J.C.,PIZZEY, J.A and PATIENT, R.K. (1994). Negative control of Xenopus GATA-2 by activin and noggin with eventual expression in precursors of the ventral blood islands. Development 120: 2519-2529.

\section{Further Related Reading, published previously in the Int. J. Dev. Biol.}

See our recent Special Issue Fertilization, in honor of David L. Garbers and edited by Paul M. Wassarman and Victor D. Vacquier at: http://www.ijdb.ehu.es/web/contents.php?vol=52\&issue=5-6

Developmentally regulated expression of hemoglobin subunits in avascular tissues Fiona C. Mansergh, Susan M. Hunter, Jenny C. Geatrell, Miguel Jarrin, Kate Powell, Martin J. Evans and Michael A. Wride Int. J. Dev. Biol. (2008) 52: 2597-2597

A change in response to Bmp signalling precedes ectodermal fate choice Chris T. Dee, Abigail Gibson, Andrea Rengifo, Shun-Kuo Sun, Roger K. Patient and Paul J. Scotting

Int. J. Dev. Biol. (2007) 51: 79-84

Expression of Bmp ligands and receptors in the developing Xenopus retina Jennifer C. Hocking and Sarah McFarlane Int. J. Dev. Biol. (2007) 51: 161-165

The expression of FIrt3 during chick limb development

Terence G. Smith and Cheryll Tickle

Int. J. Dev. Biol. (2006) 50: 701-704

BMP signalling in craniofacial development

Xuguang Nie, keijo Luukko and Paivi Kettunen

Int. J. Dev. Biol. (2006) 50: 511-521

BMP2/4 and BMP5-8 in jellyfish development and transdifferentiation

Susanne Reber-Müller, Ruth Streitwolf-Engel, Nathalie Yanze, Volker Schmid, Michael Stierwald, Michael Erb and Katja Seipel

Int. J. Dev. Biol. (2006) 50: 377-384

Cdx2 specifies the differentiation of morphological as well as functional absorptive enterocytes of the small intestine

Hiroyuki Mutoh, Kiichi Satoh, Hiroto Kita, Hirotsugu Sakamoto, Hiroko Hayakawa, Hironori Yamamoto, Norio Isoda, Kiichi Tamada, Kenichi Ido and Kentaro Sugano

Int. J. Dev. Biol. (2005) 49: 867-871

The countercurrent principle in invasion and metastasis of cancer cells. Recent insights on the roles of chemokines

Ghislain Opdenakker and Jo Van Damme

Int. J. Dev. Biol. (2004) 48: 519-527

X-epilectin: a novel epidermal fucolectin regulated by BMP signalling Karine Massé, Rebecca Baldwin, Mark W. Barnett and Elizabeth A. Jones Int. J. Dev. Biol. (2004) 48: 1119-1129

Drm/Gremlin, a BMP antagonist, defines the interbud region during feather development Boris Bardot, Laure Lecoin, Ingrid Fliniaux, Emmanuelle Huillard, Maria Marx and Jean P Viallet

Int. J. Dev. Biol. (2004) 48: 149-156

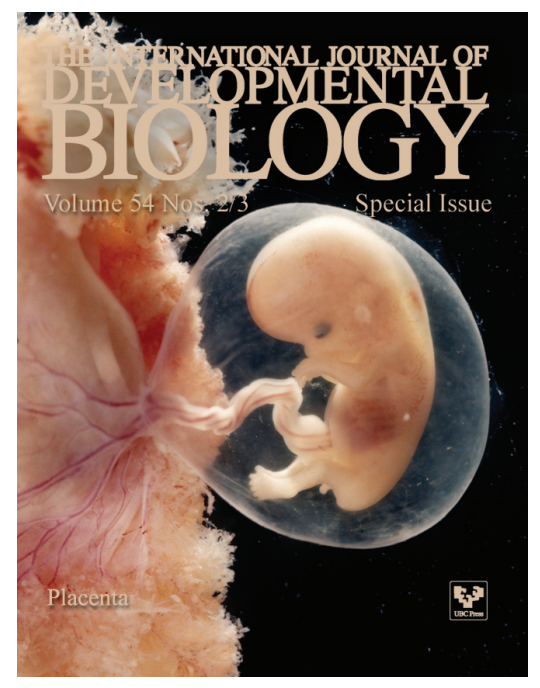

5 yr ISI Impact Factor $(2008)=3.271$

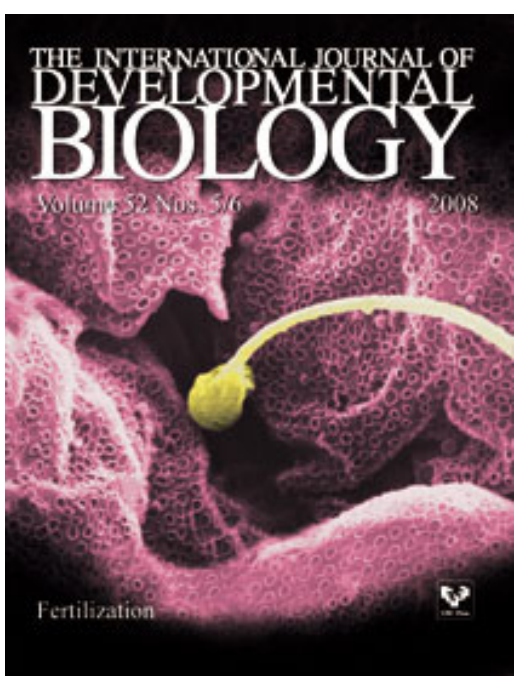

